\title{
The Relationship of Maximal Strength with the Force-Velocity Profile in Resistance Trained Women
}

\author{
Gergely Pálinkás, Bettina Béres, Zsófia Tróznai, \\ Katinka Utczás, Leonidas Petridis
}

Research Centre for Sport Physiology, University of Physical Education

Alkotás u. 44, H-1123 Budapest, Hungary

E-mails: palinkas.gergely@tf.hu, beres.bettina@tf.hu, troznai.zsofia@tf.hu, utczas.katinka@tf.hu, petridis.leonidas@tf.hu

\begin{abstract}
Force - velocity $(F-v)$ profiling is used as a diagnostic tool to evaluate ballistic performance and to optimize strength and power training. Previous research examining the lower limbs' neuromuscular qualities of female athletes has reported a large prevalence of force-deficit, which raises the question about the level of maximal strength, which may be associated with an optimal $F-v$ profile. In this study, we investigated the relationship between maximal strength abilities and the $F-v$ profile of female athletes. 27 resistancetrained female athletes (age: $29.7 \pm 5.0$ years) from three different sports (Olympic weightlifting, CrossFit and recreational bodybuilding) participated in the study. The F-v profile of the lower limbs was assessed using vertical jumps on a force platform against various external loads, while maximal strength was measured with the one-repetition maximum in the back squat. Back squat relative to body weight was calculated and used in the statistical analysis. The results showed a significant correlation between maximal back squat/body weight and the jump height during the unloaded jumps $(r=0.73 ; p<0.01)$, the theoretical maximal power $(r=0.62 ; p<0.01)$, and the ratio of the measured to the optimal $F$-v slope $(r=0.56 ; p<0.01)$. An optimal $F$-v profile was observed around $1.2 x$ bodyweight squat. Our results confirm the force-oriented profile of highly trained resistance athletes and contribute to the existing literature by providing indicative values associated with an optimal profile. Such data can be used by the coaches and athletes to evaluate the athletes' strength qualities and design training according to the individual needs.
\end{abstract}

Keywords: Olympic weightlifting; CrossFit; one repetition maximum; ballistic performance

\section{Introduction}

Force, velocity, and power are the most common measures to evaluate human muscle's mechanical properties during dynamic motor tasks. The relationship of 
force and muscle shortening velocity was first described in the early studies of Hill [1]. Based on his fundamental theorem, force and shortening velocity have an inverse relationship meaning that an increase in force causes a decrease in velocity and vica-versa. The Maximum force $\left(F_{0}\right)$ is obtained when the shortening velocity is zero (which corresponds to an isometric contraction), whereas maximum velocity $\left(v_{0}\right)$ is obtained against no external load. Additionally, by multiplying force and velocity we can calculate power. Usually, maximal power occurs at submaximal force or velocity intensities and varies between individuals. Understanding the relationship of force-velocity-power is essential in athletic training since the development of these qualities requires different training methodology.

From force and velocity measures we can define the Force-velocity $(F-v)$ slope of an athlete during ballistic movements. For lower limbs, force and velocity are typically measured using vertical jumping, more specifically, with a squat jump (SJ) or countermovement jump (CMJ), with greater power values obtained during CMJ $[2 ; 3]$. Recent studies have proposed a novel approach in the evaluation of the lower limb's main mechanical properties (force, velocity, and power) by comparing the measured slope of the force-velocity relationship $\left(\mathrm{S}_{\mathrm{fv}}\right)$ to the theoretical optimal slope $\left(\mathrm{S}_{\mathrm{fv}} \mathrm{opt}\right)[4 ; 5]$. This allows for establishing an athlete's force-velocity profile, which has been introduced as a diagnostic tool to optimize strength and power training and to monitor training adaptations [5].

The theoretical optimal $F-v$ slope represents the optimal relationship of force and velocity and is calculated by using measured indices, thus capabilities that an individual is anywise capable of. By extrapolating the regression line of the measured values we get the theoretical maximal force and the theoretical maximum velocity $\left(v_{0}\right)$, which correspond respectively to the maximum force $\left(F_{0}\right)$ and the maximum shortening velocity of the Hill-equation [1].

The percentage difference between the measured and the optimal $F-v$ slope is called force-velocity imbalance $\left(F V_{i m b}\right)$. Based on the imbalance we can quantify the relative contribution of the main components of power output (force or velocity). In strength and power training, such information may be used to determine the optimal load that is the most effective for the athletes [6], and which of the central components of power should be prioritized: force, velocity [7] or mixed methods [8; 9]. Besides the calculation of the $F V_{i m b}$, we can also calculate the theoretical maximum jump height. This represents the jump height that an athlete could reach with no changes in the strength and power qualities, but just having an optimal force-velocity relationship.

Experimental evidence has shown that $F-v$ profile is a determinative factor in ballistic, explosive movements independently of maximal power [10]. It has been proposed that at a given maximal power output, optimal $F-v$ profile maximizes performance, whereas an imbalance in force-velocity profile $\left(F V_{\text {imb }}\right)$ can cause $\sim 30 \%$ decrease in performance [10] and negatively affects ballistic performance 
[4]. As suggested, $F-v$ profile can be a better indicator to describe the power capabilities of an athlete than jump height [11].

Athletes with notable $F V_{i m b}$ are suggested to first decrease the imbalance and then target further development in maximal power [12]. The type of training to reduce the imbalance is individual and depends on the magnitude and the direction of the $F V_{\text {imb }}$.

Available $F$-v profile data with female athletes have reported mostly force-deficit. For example, Marcote-Pequeno et al.[13] using squat jump and not CMJ, found $F V_{i m b}$ of $64.5 \pm 16.3 \%$ of force-deficit in female soccer athletes. The $F-v$ profile of elite handball and volleyball players [14] revealed $F V_{i m b}$ values of $31.1 \pm 14.0 \%$ and $22.1 \pm 9.6 \%$ of again mainly force-deficit. Such results raise the question of the level of maximal strength, which may be linked to an optimal $F-v$ profile in female athletes. The only study examining the relationship of the $F$ - $v$ profile for the lower limbs with the maximal strength describes the positioning of the onerepetition maximum (1RM) on the force-velocity curve [15]. As expected, the $1 \mathrm{RM}$ is positioned below the theoretical maximal force $\left(F_{0}\right)$ and above the force exerted against the heaviest load during the loaded jumps, which seems reasonable since $F_{0}$ represents the maximal force under isometric conditions (when velocity is zero) and in general is larger than the 1RM. However, this is a theoretical approach with limited transferability to coaching practice.

From a practical point of view, it would be useful to examine the connection of $F-v$ profile with the maximal strength as measured with the one-repetition maximum. 1RM is a quite common measure of maximal strength in everyday practice and its assessment does not require expensive equipment. The results can be expressed either in absolute or relative to bodyweight values making comparisons possible between athletes of different body weights. Due to its similar movement pattern with vertical jump and to its close connection with the lower limbs' power production ability, back squat exercise is widely used for strength training and testing purposes [16]. Such results could help to evaluate the maximal strength capabilities providing a better understanding of the athletes' strength qualities. In turn, this may support the strength training of the athletes, not only in traditional strength-based sports but also in team sports [17; 18], where our earlier findings showed considerable force deficit in Hungarian elite female athletes [14]. While force-oriented training uses mostly heavy resistance loads targeting the force-end of the force-velocity curve, velocity oriented training uses light (or no external) resistance loads targeting the velocity-end of the forcevelocity curve. In power training, it has been suggested that weaker athletes can benefit from improving strength (use of heavier loads), while stronger athletes can benefit from a more specialized, ballistic type training [19]. Overall, a mixed approach is recommended, which encompasses various loads affecting the entire range of the force-velocity spectrum. 
Therefore, the purpose of this study was to examine the relationship of the lower limbs' Force-velocity profile with the relative to bodyweight maximal back squat in resistance-trained women. It was hypothesised that maximal performance in back squat correlates with the $F-v$ profile with higher strength levels being associated with a force-oriented profile.

\section{Material and Methods}

\subsection{Participants}

27 resistance-trained female athletes (age: $29.7 \pm 5.0$ years; body height: $166.7 \pm$ $4.6 \mathrm{~cm}$, bodyweight: $66.0 \pm 8.2 \mathrm{~kg}$; training load: $5.9 \pm 2.4$ hours/week; training history: $2.5 \pm 1.8$ years) from three different sports (Olympic weightlifting, CrossFit and recreational bodybuilding) and different competition levels (intermediate to international level) with a minimum of one year experience in strength training participated in the study. None of the subjects reported illness or injury at the time of the measurements. All participants were informed about the type and the risks of the measurements and gave their written consent to participate in this study. The study was approved by the University's Research Ethics Committee (approval number: TE-KEB/NO34/2019).

\subsection{Measurements}

Measurements took place at the laboratory of the University of Physical Education Budapest. After arrival, participants went through basic anthropometric measurements. Body height was measured with a stadiometer to the nearest 0.1 $\mathrm{cm}$. Bodyweight (BW) was measured with a digital scale (Seca 888) to the nearest $0.1 \mathrm{~kg}$.

A vertical jump test was performed on a force platform (FP4, HUR Labs Oy, Tampere, Finland) with a sampling rate of $1000 \mathrm{~Hz}$. First, subjects performed a unified warm-up protocol which contained mobilization exercises and running. Then, they performed five submaximal countermovement jumps (CMJ) to ensure proper technique. Participants performed three unloaded jumps, then two loaded jumps against external loads of 25, 50, 75, and 100 percent of their BW. During unloaded jumps hands were kept on the hips, while during loaded jumps on the bar. Loaded jumps were performed with free weights. The rest time between jumps was two minutes to ensure total recovery. In this study, the best jumps were used in the statistical analysis. Participants were asked to refrain from any exercise 24 hours prior to the measurements. 
A one-repetition maximum (1RM) full back squat protocol was used to assess maximal strength of the lower limbs. Measurements protocol consisted of two sets of three repetitions at 50, one set of two repetitions at 70 then one set of one repetition at 80,90 , and 95 percent of their previous $1 \mathrm{RM}$ back squat. Then, they attempted to hit the maximum weight they can squat with. Rest time was two minutes between sets. Full squats were performed with a high bar technique. To accept a proper technique of full squat, the subjects' hip had to go lower than the horizontal line of the knees and they had to be able to hold the bar without losing tension of the upper body. Testing of the 1RM back squat was performed 72 hours before the jumping protocol.

\subsection{Calculations}

Mean force values for every loaded condition were extracted from the force platform during the push-off phase (when ground reaction force becomes greater than the bodyweight to the instant of takeoff). Push off distance (hpo) was defined as the vertical displacement of the centre of mass (COM) moved from the lowest point of the countermovement to the instant of takeoff and it was extracted from the vertical displacement-time curve of the force platform. For the calculation of the jump height, we used the impulse-momentum method [20].

The average speed of the jumping movement was calculated from jump height according to [5]:

$v=\sqrt{\frac{9.81 * \text { Jump Height }}{2}}$

where $v$ is the average speed of the jump, jump height is the height of the jump based on the impulse-momentum method.

The theoretical maximal power $\left(P_{\max }\right)$ was calculated from the theoretical maximal force $\left(F_{0}\right)$ and the theoretical maximal velocity $\left(v_{0}\right)$ according to the following formula [21]:

$P_{\max }=\frac{F_{0} * v_{0}}{4}$

Based on the $P_{\max }$ and $h_{p o}$ values, we calculated the optimal slope $\left(\mathrm{Nskg}^{-1} \mathrm{~m}^{-1}\right)$ for force and velocity. Once we have the optimal and the measured slope, $F-v$ imbalance can be computed based on the following formula [10]:

$F V_{i m b}=100 *\left|1-\frac{S_{f v}}{S_{f v} o p t}\right|$

where $F V_{i m b}$ is the difference (in $\left.\%\right)$ between optimal $\left(S_{f v} o p t\right)$ and measured $\left(S_{f v}\right)$ slope of the force-velocity relationship. 
The theoretical maximum height $\left(h_{\max }\right)$ of the vertical jump was calculated based on $S_{f v o p t}$ with no changes in $P_{\max }$ and $h_{p o}$ values. We used the following recently validated formula to calculate $h_{\max }[10]$ :

$h_{\text {max }}=\frac{h_{P O}{ }^{2}}{2 g}\left(\sqrt{\frac{S_{f v} o p t^{2}}{4}+\frac{2}{h_{P O}}\left(\sqrt{2-P_{\max } * S_{f v} o p t}-g\right)}+\frac{S_{f v} o p t}{2}\right)^{2}$

where $h_{p o}$ is the height of push-off phase, $S_{f v}$ opt is the optimal slope of the Forcevelocity curve, $P_{\max }$ is the theoretical maximal power, $g$ is the acceleration due to gravity.

\subsection{Statistical Analysis}

Values are expressed as means \pm SD. Intraclass correlation coefficient (ICC) was used to assess reliability between jumps in each loaded condition. Shapiro-Wilks test was used to assess normality. Outliers for vertical jump height were removed under P25-1.5 * (P75-P25) or above P75+1.5* (P75-P25) value, where P25 is the 25th and P75 is the 75th percentile. Pearson's correlation was used to quantify the correlation between relative back squat and power, jump height, and $F V_{\text {imb }}$. Correlation coefficients were classified as 0.0-0.1 (trivial); 0.1-0.3 (small); 0.3-0.5 (moderate); 0.5-0.7 (large); 0.7-0.9 (very large); 0.9-1.0 (near perfect) [22]. Statistica 13.5 for windows (TIBCO Software Inc, Palo Alto, CA, USA) statistical package was used for the statistical analysis.

\section{Results}

Relative values of the subjects in back squat 1RM showed normal distribution. ICC was 0.95 (95\% CI 0.90 to 0.98 ) for unloaded jumps, 0.99 (95\% CI 0.96 to 0.99 ) for loaded jumps with $25 \%$ of BW, 0.97 (95\%CI 0.92 to 0.99 ) with $50 \%$ of BW, 0.99 (95\% CI 0.97 to 0.99 ) with $75 \%$ of BW and 0.97 (95\% CI 0.92 to 0.99 ) with $100 \%$ of BW, respectively. The descriptive results for the unloaded CMJ and for the $F-v$ profile can be found in tables 1 and 2, respectively.

Table 1

Descriptive results for the unloaded countermovement jump

\begin{tabular}{|l|cccc|}
\hline Variable & mean & SD & min & max \\
\hline Jump Height (cm) & 29.0 & 4.8 & 19.9 & 43.1 \\
Push off distance (m) & 0.37 & 0.04 & 0.28 & 0.43 \\
Average Force abs. (N) & 867.6 & 108.4 & 642.3 & 1034.5 \\
Average Force rel. (N/kg) & 13.2 & 0.5 & 12.4 & 14.5 \\
Average Power abs. (W) & 777 & 126 & 576 & 1074 \\
Average Power rel. (W/kg) & 11.8 & 1.6 & 8.7 & 15.3 \\
\hline
\end{tabular}

abs. $=$ absolute values, rel.$=$ relative values 
Table 2

Descriptive results of the Force-velocity profile

\begin{tabular}{|l|cccc|}
\hline Variable & mean & SD & min & max \\
\hline Theoretical $F_{0}(\mathrm{~N} / \mathrm{kg})$ & 35.58 & 5.67 & 23.80 & 46.33 \\
Theoretical $v_{0}(\mathrm{~m} / \mathrm{s})$ & 2.47 & 0.36 & 1.90 & 3.70 \\
Theoretical jump height $(\mathrm{m})$ & 0.31 & 0.05 & 0.21 & 0.43 \\
Measured $F-v$ slope & -14.84 & 3.65 & -22.06 & -6.84 \\
$(\mathrm{Ns} / \mathrm{m} / \mathrm{kg})$ & -14.66 & 0.74 & -16.89 & -13.51 \\
Optimal $F-v$ slope $(\mathrm{Ns} / \mathrm{m} / \mathrm{kg})$ & 20.1 & 14.9 & 0.3 & 55.2 \\
$F-v$ imbalance $(\%)$ &
\end{tabular}

$F_{0}$ : maximal force; $v_{0}$ : maximal velocity; $F-v$ slope: slope of the force-velocity relationship; $F-v$ imbalance: force-velocity imbalance

$F V_{i m b}$ values showed velocity deficit in 12 cases (44\%) and force deficit in 15 (55\%) cases. $22 \%$ of the subjects (6 athletes) had normal (less than 10\%), 59\% (16 athletes) had low (between 10\% and 40\%) and 19\% (5 athletes) had high (greater than $40 \%$ ) imbalance. On average, measured jump height corresponded to $94 \%$ of the theoretical maximum. Three subjects' jump height was greater than their theoretical maximum.

A strong correlation was found between the back squat and weekly training load $(\mathrm{r}=0.86 ; \mathrm{p}<0.05)$, while the correlation between back squat and training history of the subjects was moderate to large $(r=0.69 ; \mathrm{p}<0.05)$.

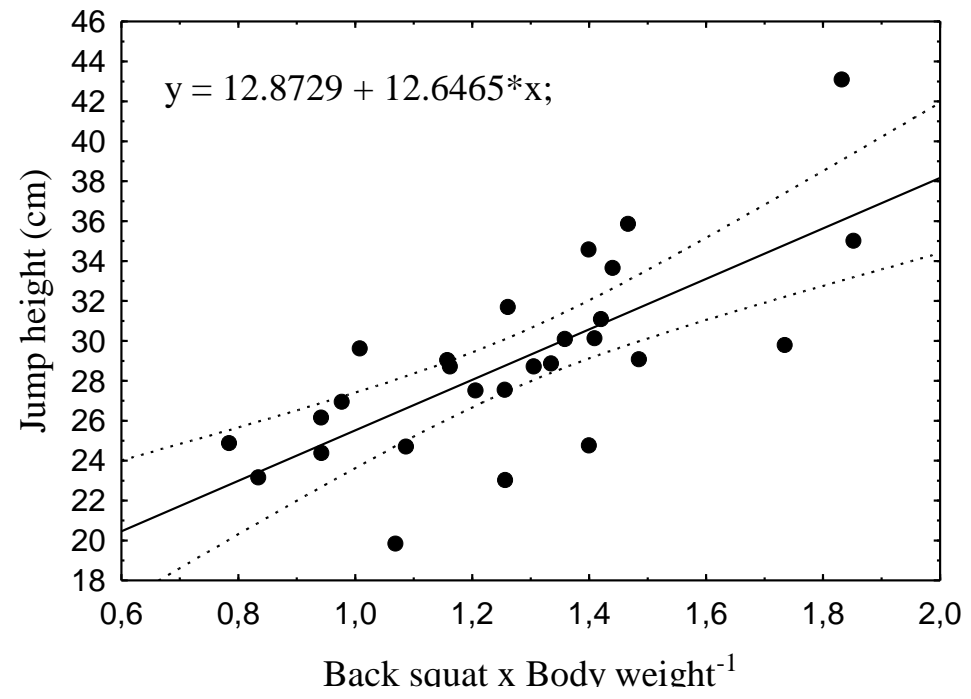

Figure 1

Correlation between the jump height during unloaded jumps and the relative values of one repetition maximum back squat. Dotted lines represent $95 \%$ CI. 
Figures 1, 2, and 3 show the significant correlations between the relative strength and the height of the unloaded jumps, $(\mathrm{r}=0.73 ; \mathrm{p}<0.01)$, the $P_{\max }(\mathrm{r}=0.62 ; \mathrm{p}<0.01)$, and the ratio of the measured to optimal $F-v$ slope $(\mathrm{r}=0.56 ; \mathrm{p}<0.01)$. Subjects with better back squat*body weight ${ }^{-1}$ ratio reached higher values during the jumps. Optimal $F V_{\text {imb }}$ values can be found around the 1.2 value of back squat * body weight $^{-1}$.

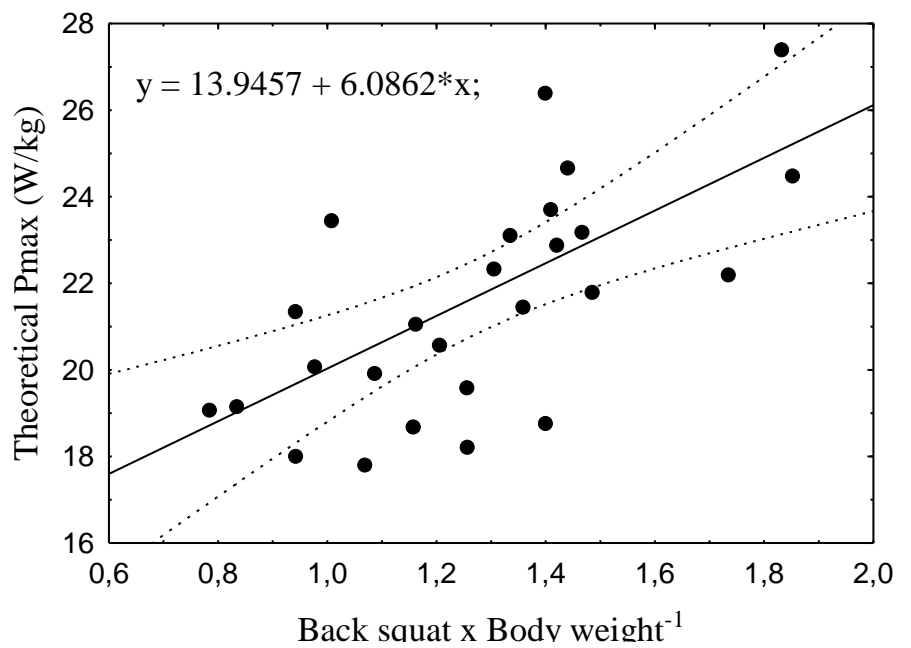

Figure 2

Correlation between the theoretical maximal Power and the relative values of one repetition maximum back squat. Dotted lines represent $95 \%$ CI.

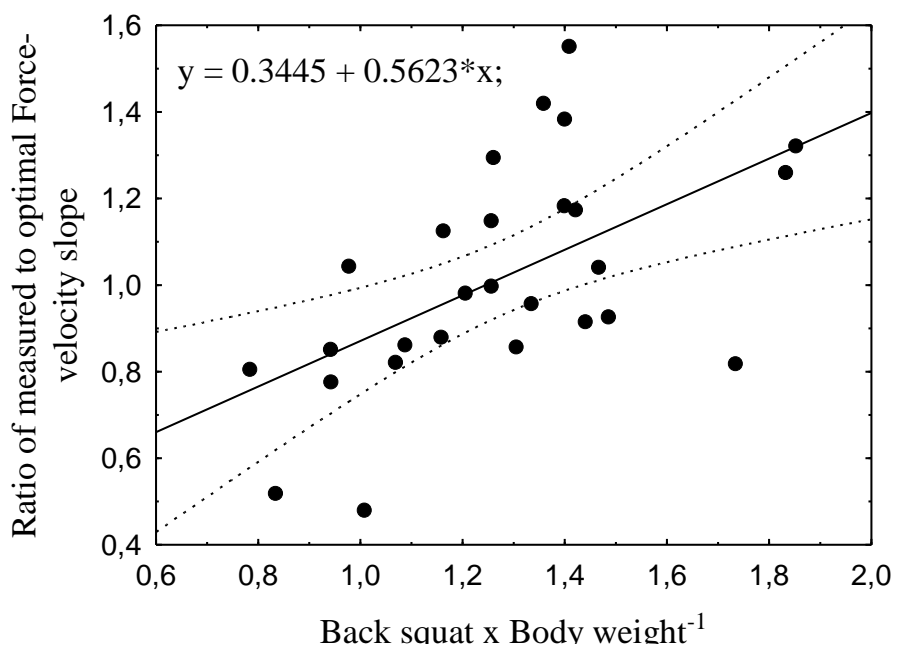

Figure 3

Correlation between ratio of the measured to the optimal Force-velocity slope and the relative values of one repetition maximum back squat. Dotted lines represent $95 \%$ CI. 


\section{Discussion}

The aim of this study was to investigate the relationship between maximal strength abilities and the $F-v$ profile in resistance-trained female athletes, attempting to determine the minimum strength requirements for an optimal profile. $F-v$ profiling has gained attention as a diagnostic measurement by separating the strength and velocity abilities of an athlete and describing training directions according to the individual needs.

Previous studies with female athletes have reported $F-v$ profile of mainly forcedeficit $[13 ; 14]$. Force-deficit indicates the need for strength-oriented training, which targets the force-end of the force-velocity spectrum [19]. This is supported by studies showing that the type of strength training has a direct effect on the force-velocity qualities and that the $F-v$ profile reflects adaptations in strength training $[12 ; 23]$. Generally, resistance-trained athletes usually demonstrate a force-oriented profile, while athletes not used to heavy loads training have a velocity-oriented profile $[10 ; 12 ; 24]$. Being used to resistance training with heavy loads the proportion of the athletes in our sample, who had a low to high force deficit was $48 \%$, which is considerably lower than the same proportion found in our previous study with elite handball and volleyball players (83.3\%) [14]. The typical force-oriented profiles of resistance-trained athletes were demonstrated also in the study of Jimenez-Reyes et al. [25] with athletes from different sports and competition levels. Olympic weightlifters had significantly higher $F_{0}$ values than elite athletes from team sports (soccer, handball), indicating the differences in the mechanical attributes of the lower limbs in sports of different movement patterns and physical requirements [25]. The weightlifters in our study had similar $F_{0}$ and $v_{0}$ values to high-level weightlifters and high-level track \& field athletes reported by Jimenez-Reyes et al. [25]. While $F_{0}$ values (38.0 $\pm 7.3 \mathrm{~N} / \mathrm{kg})$ were higher in weightlifters in both studies, $v_{0}$ values $(2.6 \pm 0.1 \mathrm{~m} / \mathrm{s})$ seem to be within the average range consisting of several sports as represented in the study of Jimenez-Reyes et al. [25]. CrossFit athletes had lower $F_{0}$ values (35.4 $\pm 5.7 \mathrm{~N} / \mathrm{kg})$ with a lower $v_{0}(2.5 \pm 0.4 \mathrm{~m} / \mathrm{s})$ than weightlifters. Both groups produced greater forces, than athletes from non-strength-based sports, confirming the force-oriented profiles resulting from resistance training.

The larger prevalence of force deficit in previous studies highlights the importance of maximal strength in power training. In relation to maximal strength, an observation and a question emerge. As expected, and confirming existing knowledge [16], maximal strength is a determinant factor in performance during ballistic movements. This was well reflected in the correlation analysis. Relative values in 1RM back squat positively correlated with the performance in vertical jumping (Figure 1) and with the theoretical maximal power output (Figure 2). Athletes with higher relative to bodyweight back squat achieved higher jump height and had a higher theoretical maximal power. These results clearly reinforce the significance of maximal strength training to increase vertical jump performance. 
However, the question, which is of great interest for coaches and practitioners appertains to the levels of maximal strength which may result in an optimal profile. How strong an athlete should be in order to prevent force deficit? Haff and Nimphius [19] have suggested that strong athletes must be able to squat with two times their bodyweight. However, this is a general recommendation and does not discriminate between men and women or in relation to different bodyweight categories. With regard to the $F-v$ profile, these values need to be examined experimentally separately for male and female athletes. In our sample with female athletes and according to our expectations, the statistical analysis revealed a positive, large correlation between relative 1RM back squat and the ratio of optimal to the measured $F-v$ slope. The findings suggest that the optimal range of the $F-v$ profile is associated with a relative 1RM back squat around $1.2^{*}$ of body weight. Athletes with greater relative values show force-oriented, while lower values show mostly velocity-oriented profiles. This value is significantly lower than the recommendations of a back squat with $2.0 *$ of body weight. Although significant, the magnitude of the correlation raises several questions concerning the conclusive use of $F-v$ profile results from 1RM in the back squat.

Two aspects should be considered in the interpretation of these results. First, the robustness of the measurements. The 1RM measurement is a robust measurement showing little variations within a microcycle [26]. Meaningful changes in 1RM are usually observed after greater time periods, a minimum of four weeks of strength training has been reported in the literature [27]. In the contrary, we are unaware of any studies examining the consistency of $F-v$ profiling across consecutive days. However, based on our experience, it seems that $F$ - $v$ profiling is more sensitive to variations within a training microcycle and may depend more on the training load on the days preceding the measurements. While acknowledging the need to confirm this experimentally, the sensitivity of these measurements to changes in the instantaneous training status of the athletes may have affected the correlation statistical analysis.

A second aspect to be considered relates to the limitations of the extrapolated $F$ - $v$ relationship. Measured points on the $F-v$ curve cover only about $30 \%$ of the velocity values [15]. Mean velocity at the heaviest load during vertical jumping was on average $1.46 \mathrm{~m} / \mathrm{s}$. Conversely, the velocity usually reached during $1 \mathrm{RM}$ exercise in female athletes is around $\sim 0.2 \mathrm{~m} / \mathrm{s}$, which is less by about $700 \%$, than during $F-v$ profile assessment [28]. Thus, exercise at 1RM is a much slower movement than during vertical jumping. In this sense, these two measurements demonstrate different kinematic profiles and indicate different qualities in strength training. Moreover, and according to Riviere et al. [15], the force developed during the $1 \mathrm{RM}$ measurement is about $16 \pm 4 \%$ higher than the force during the jump against the heaviest load. Given, that Riviere et al. [15] used a half squat $1 \mathrm{RM}$ measurement we assume that the force developed during a full squat as applied in our study is higher and therefore less close to the force measured during the jump against the heaviest load. In other words, relatively large differences 
exist between the maximum forces developed during the two measurements. This suggests that when examining the relationship between 1RM and $F-v$ profile during vertical jumps maximum loads should exceed $100 \%$ of $\mathrm{BW}$, hence being closer to the 1RM force exertion.

\section{Conclusions}

While force-velocity profiling is a well-known tool in performance diagnostics, our study contributes to the existing literature by examining the relationship of the $F$-v profile during vertical jumps with the relative 1RM in the back squat. Such an approach aims at determining the minimal requirements in strength in order to achieve an optimal profile. The results (1) confirm the force-oriented profiles of resistance-trained athletes and (2) suggest that female athletes with a ratio of 1.2 back squat $*$ bodyweight ${ }^{-1}$ can achieve an optimal $F-v$ profile. Such data can be used by coaches and researchers as benchmark values in the evaluation of the athletes' strength qualities. They can provide a reference point to design training interventions based on the one-repetition maximum values by determining the main directions in strength training.

\section{Acknowledgements}

The authors would like to thank all athletes who participated in our study. We are grateful to Dr. Tamás Szabó (University of Physical Education) for his substantial contribution in the design and formation of our laboratory allowing the conduction of such measurements. Also, to Balázs Farkas, head coach of CrossFit Mayfly Budapest, who provided the venue to conduct part of the measurements.

\section{References}

[1] A. Hill, The heat of shortening and the dynamic constants of muscle, P. Roy. Soc. B-Biol. Sci., 1938, pp. 136-195

[2] P. Jimenez-Reyes, P. Samozino, V. Cuadrado-Penafiel, F. Conceicao, J. Gonzalez-Badillo, J. Morin, Effect of countermovement on power-forcevelocity profile. European Journal of Applied Physiology 114 (2014) 2281 2288

[3] P. Jimenez-Reyes, P. Samozino, F. Pareja-Blanco, F. Conceicao, V. Cuadrado-Penafiel, J. Gonzalez-Badillo, J. Morin, Validity of a Simple Method for Measuring Force-Velocity-Power Profile in Countermovement Jump. International Journal of Sports Physiology and Performance 12 (2017) 36-43

[4] J. Morin, P. Samozino, Interpreting Power-Force-Velocity Profiles for Individualized and Specific Training. International Journal of Sports Physiology and Performance 11 (2016) 267-272

[5] P. Samozino, J. Morin, F. Hintzy, A. Belli, A simple method for measuring force, velocity and power output during squat jump. Journal of Biomechanics 41 (2008) 2940-2945 
[6] D. Behm, J. Young, J. Whitten, J. Reid, P. Quigley, J. Low, Y. Li, C. Lima, D. Hodgson, A. Chaouachi, O. Prieske, U. Granacher, Effectiveness of Traditional Strength vs. Power Training on Muscle Strength, Power and Speed with Youth: A Systematic Review and Meta-Analysis. Frontiers in Physiology 8:423 (2017)

[7] N. Kawamori, R. Newton, Velocity specificity of resistance training: Actual movement velocity versus intention to move explosively. Strength and Conditioning Journal 28 (2006) 86-91

[8] G. Duthie, W. Young, D. Aitken, The acute effects of heavy loads on jump squat performance: An evaluation of the complex and contrast methods of power development. Journal of Strength and Conditioning Research 16 (2002) 530-538

[9] N. Elbadry, A. Hamza, P. Pietraszewski, D. Alexe, G. Lupu, Effect of the French Contrast Method on Explosive Strength and Kinematic Parameters of the Triple Jump Among Female College Athletes. Journal of Human Kinetics 69 (2019) 225-230

[10] P. Samozino, E. Rejc, P. Di Prampero, A. Belli, J. Morin, Optimal ForceVelocity Profile in Ballistic Movements-Altius: Citius or Fortius? Medicine and Science in Sports and Exercise 44 (2012) 313-322

[11] J. Morin, P. Jimenez-Reyes, M. Brughelli, P. Samozino, When Jump Height is not a Good Indicator of Lower Limb Maximal Power Output: Theoretical Demonstration, Experimental Evidence and Practical Solutions. Sports Medicine 49 (2019) 999-1006

[12] P. Jimenez-Reyes, P. Samozino, J. Morin, Optimized training for jumping performance using the force-velocity imbalance: Individual adaptation kinetics. Plos One 14 (2019)

[13] R. Marcote-Pequeno, A. Garcia-Ramos, V. Cuadrado-Penafiel, J. Gonzalez-Hernandez, M. Gomez, P. Jimenez-Reyes, Association Between the Force-Velocity Profile and Performance Variables Obtained in Jumping and Sprinting in Elite Female Soccer Players. International Journal of Sports Physiology and Performance 14 (2019) 209-215

[14] L. Petridis, G. Pálinkás, Z. Tróznai, B. Béres, K. Utczás, Determining strength training needs using the force-velocity profile of elite female handball and volleyball players. International Journal of Sport Science \& Coaching (2021) 123-130

[15] J. Riviere, J. Rossi, P. Jimenez-Reyes, J. Morin, P. Samozino, Where does the One-Repetition Maximum Exist on the Force-Velocity Relationship in Squat? International Journal of Sports Medicine 38 (2017) 1035-1043

[16] K. Wirth, H. Hartmann, A. Sander, C. Mickel, E. Szilvas, M. Keiner, The Impact of Back Squat and Leg-Press Exercises on Maximal Strength and 
Speed-Strength Parameters. Journal of Strength and Conditioning Research 30 (2016) 1205-1212

[17] M. Hammami, N. Gaamouri, G. Aloui, R. Shephard, M. Chelly, Effects of a Complex Strength-Training Program on Athletic Performance of Junior Female Handball Players. International Journal of Sports Physiology and Performance 14 (2019) 163-169

[18] L. Rodriguez-Lorenzo, M. Fernandez-Del-Olmo, R. Martin-Acero, Strength and Kicking Performance in Soccer: A Review. Strength and Conditioning Journal 38 (2016) 106-116

[19] G. Haff, S. Nimphius, Training Principles for Power. Strength and Conditioning Journal 34 (2012) 2-12

[20] N. Linthorne, Analysis of standing vertical jumps using a force platform. American Journal of Physics 69 (2001) 1198-1204

[21] H. Vandewalle, G. Peres, H. Monod, STANDARD ANAEROBIC EXERCISE TESTS. Sports Medicine 4 (1987) 268-289

[22] W. Hopkins, S. Marshall, A. Batterham, J. Hanin, Progressive Statistics for Studies in Sports Medicine and Exercise Science. Medicine and Science in Sports and Exercise 41 (2009) 3-12

[23] P. Jimenez-Reyes, P. Samozino, M. Brughelli, J. Morin, Effectiveness of an Individualized Training Based on Force-Velocity Profiling during Jumping. Frontiers in Physiology 7 (2017)

[24] P. Jimenez-Reyes, M. Cross, A. Ross, P. Samozino, M. Brughelli, N. Gill, J. Morin, Changes in mechanical properties of sprinting during repeated sprint in elite rugby sevens athletes. European Journal of Sport Science 19 (2019) 585-594

[25] P. Jimenez-Reyes, P. Sarnozino, A. Garcia-Ramos, V. Cuadrado-Penafiel, M. Brughelli, J. Morin, Relationship between vertical and horizontal forcevelocity-power profiles in various sports and levels of practice. Peerj 6 (2018)

[26] P. Comfort, J. McMahon, RELIABILITY OF MAXIMAL BACK SQUAT AND POWER CLEAN PERFORMANCES IN INEXPERIENCED ATHLETES. Journal of Strength and Conditioning Research 29 (2015) 3089-3096

[27] T. Freitas, A. Martinez-Rodriguez, J. Calleja-Gonzalez, P. Alcaraz, Shortterm adaptations following Complex Training in team-sports: A metaanalysis. Plos One 12 (2017)

[28] A. Torrejon, C. Balsalobre-Fernandez, G. Haff, A. Garcia-Ramos, The load-velocity profile differs more between men and women than between individuals with different strength levels. Sports Biomechanics 18 (2019) 245-255 